\title{
Pharmacy Benefit Forecast for a New Interferon Beta-1a for the Treatment of Multiple Sclerosis: Development of a First-Line Decision Tool for Pharmacy-Budget Planning Using Administrative Claims Data
}

CHRISTINA M. MEYER, MHS; ROBERT PHIPPS, PharmD; DEBORAH COOPER, PharmD, BCPS; and ALAN WRIGHT, MD, MPH

\begin{abstract}
OBJECTIVE: To estimate the incremental change in pharmacy per-member-permonth (PMPM) costs, according to various formulary designs, for a new interferon beta-1a product (IB1a2) using administrative claims data.

METHODS: Cross-sectional sex- and age-specific disease prevalence and treatment rates for relapsing, remitting multiple sclerosis (RRMS) patients were measured using integrated medical and pharmacy claims data from a 500,000member employer group in the southern United States. Migration to IB1a2 from other drugs in the class was based on market-share data for new and existing RRMS patients. Duration of therapy was estimated by analyzing claims for current RRMS therapies. Daily therapy cost was provided by the manufacturer of IB1a2, adjusted for migration from other therapies, and multiplied by estimated volume to predict incremental and total PMPM cost impact. Market-share estimates were used to develop a PMPM cost forecast for the next 2 years. PMPM cost estimates were calculated for preferred (copayment tier 2) and nonpreferred (copayment tier 3) formulary designs with and without prior authorization (PA). One-way sensitivity analysis was performed to assess the influence of product pricing, duration of therapy, and other market factors.
\end{abstract}

RESULTS: Annual incremental PMPM change was $\$ 0.047$ for the scenario of third copayment tier with PA. The incremental change was greatest for those aged 55 to 65 years ( $\$ 0.056$ PMPM) and did not vary greatly by benefit design. Duration of therapy had the greatest impact on the PMPM estimate across benefit designs.

CONCLUSION: IB1a2 will not cause a significant change in managed care pharmacy budgets under a variety of formulary conditions, according to this crosssectional analysis of current care-seeking behavior by RRMS patients. Economic impact may differ if IB1a2 expands RRMS patients' treatment-seeking behavior.

KEYWORDS: Interferon beta-1a, Multiple sclerosis, Treatment costs

\section{J Managed Care Pharm. 2003(9)2:168-74}

\section{Authors}

CHRISTINA M. MEYER, MHS, is Manager, Predictive Modeling and Forecasting; DEBORAH COOPER, PharmD, BCPS, is Director, Pharmaceutical Impact and Trends; and ALAN WRIGHT, MD, MPH, is Chief Science Officer, AdvancePCS, Hunt Valley, Maryland; ROBERT PHIPPS, PharmD, is Vice President, Managed Markets, Serono Inc., Rockland, Massachusetts.

AUTHOR CORRESPONDENCE: Christina M. Meyer, MHS, Manager, Predictive Modeling and Forecasting, AdvancePCS, 11350 McCormick Rd. Executive Plaza II, Suite 1000, Hunt Valley, MD 21031. Tel : (410) 785-2146; Fax: (410) 785-8140; E-mail: Christina.Meyer@AdvancePCS.com

Copyright $\odot$ 2003, Academy of Managed Care Pharmacy. All rights reserved.
A new form of interferon beta-la (IB1a2) was approved in March 2002 for the treatment of relapsing, remitting multiple sclerosis (RRMS). ${ }^{1}$ Clinical trial results suggested improved clinical efficacy compared to the previous formulation of interferon beta-la (IBla1), leading to an override of the existing product's orphan-drug-status exclusivity. ${ }^{2}$ IBla2, an injectable, subcutaneous therapy, will be a direct competitor in the RRMS marketplace, ${ }^{3}$ which includes IBlal, interferon betalb (IBlb), and glatiramer (Table 1). As a new first-line therapy and expensive biotech product, health plans may need to consider the pharmacy-budget impact of this product.

RRMS is the most common presentation of multiple sclerosis at onset, affecting $65 \%$ to $85 \%$ of newly diagnosed cases. ${ }^{4,5}$ Although therapies attempt to slow progression, many RRMS patients develop the secondary progressive (SPMS) form of the disease and face significant disability. ${ }^{4}$ Some new MS cases may present in the primary progressive form $(10 \%)$ without relapses or remissions in symptoms over time. ${ }^{5}$ Fewer cases (5\%) have the primary relapsing form, which is characterized by chronic deterioration and acute episodes. ${ }^{5}$ Managed care prevalence of MS in insured populations has been measured at rates of 24/10,000 enrollees for privately insured populations, with higher rates in Medicare $(36 / 10,000)$ and Medicaid $(71 / 10,000)$ over 2 years. ${ }^{6}$

This benefit forecast analysis quantifies pharmacy costs from the perspective of the managed care payer, using actual careseeking behavior as measured in administrative claims. It does not consider literature-based values, such as quality of life or medical-cost offsets, as do traditional cost-effectiveness or cost-utility models that may not reflect actual community practice. ${ }^{7-9}$ The forecasting analysis quantifies the use of therapies in a population and calculates the budget impact based on a simple scenario: unit cost multiplied by volume.

The importance of this approach is that it provides timely data regarding anticipated (pipeline) or recently approved therapies that may concern decision makers because of price, potential adoption as a new standard of care, or the number of patients affected.

Indeed budget impact represents an additional hurdle in the drug uptake process for managed care organizations after consideration of other primary factors, including safety, efficacy, and quality. The proposed technique addresses the pharmacy 


\section{TABLE $1 \longdiv { \text { Study Drugs } }$}

\begin{tabular}{lllllll} 
Generic Name & Abbreviation & Brand Name & Manufacturer & Form & Strength & Administration \\
\hline Interferon beta-la & IBlal & Avonex & Biogen & Powder; injection kit & 30 mcg & Once weekly, IM \\
\hline Interferon beta-la & IBla2 & Rebif & Serono SA & Prefilled syringe & $22 \mathrm{mcg}, 44 \mathrm{mcg}$ & $3 \mathrm{x}$ weekly, SC \\
\hline Interferon beta-lb & IBlb & Betaseron & Berlex & Powder; injection kit & $0.25 \mathrm{mg}$ & QOD, SC \\
\hline Glatiramer acetate & Glatiramer & Copaxone & Teva Marion & $\begin{array}{l}\text { Prefilled syringe and } \\
\text { powder; injection kit }\end{array}$ & $20 \mathrm{mg}$ & QD, SC \\
\hline
\end{tabular}

Sources: Physicians' Desk Reference, 54th ed., 2002; Rebif product information (Serono Laboratories, Inc., 2002); and Copaxone product information, Teva Neurosciences, 2002.

budget impact as it affects administrators determining coverage decisions. Actual value of the therapy in relation to its competitors would need to be measured by cost-effectiveness analysis that incorporates safety and efficacy outcomes. While IBla2 may be clinically superior to its competitors in clinical trials, a cost-effectiveness analysis would be needed to show long-term benefit in community use. These end points may not be immediately relevant to payer decisions about costs in the short-term, which necessitates budget impact analyses.

When budget impact is small, formulary decision makers can focus on safety and efficacy differences among products. In contrast, when budget impact is high, the situation may be complicated by conflicting consumer, physician, and payer demands. Payers may then want to shift focus to more extensive cost-effectiveness or cost-benefit studies. In these cases, establishing cost offsets on the medical side may be necessary to justify the pharmacy-budget impact.

We conducted this study to estimate the pharmacy-budget cost impact of IBla2, a new RRMS treatment. The analysis emphasized the following attributes in its design: transparency to the managed care audience, usage estimates based on actual care-seeking behavior in a managed care setting, sensitivity analysis of key uncertainties, a short-term time horizon (1-3 years), and simplicity of relevant metrics for decision-makers using per-member-per-month (PMPM) cost estimates.

\section{Study Design and Methods}

A cost-identification analysis of pharmacy-budget impact using integrated medical and pharmacy claims data was automated into a sensitivity-analysis model that can be customized to accommodate data from different health plans, benefit designs, and market scenarios.

Several assumptions were made regarding the anticipated usage of IBla2 and the market for RRMS treatments:

- The RRMS-treatment market is saturated.

- There will be no expansion of the market once interferon beta la is launched.

- There will be no off-label use of this injectable therapy.

- IBla2 will compete directly with leading market contenders IBlal, IBlb, and glatiramer.

- IB1a2 will be used as first-line therapy for RRMS.
- IB1a2 patient compliance with therapy may increase due to a better side-effect profile.

\section{Identification of Base-Case Model Population}

A 500,000-member, state-employer group in the southern United States was selected as the test plan to define model inputs using integrated medical and pharmacy claims data for calendar year 2000. This plan provided pharmacy coverage for members aged 65 years and older as part of a major medical supplement to Medicare; i.e., these are not Medicare+Choice members. The portion of the population covered under the State Children's Health Insurance Program (SCHIP) was eliminated from the analysis. ${ }^{10}$

Rates of MS were described separately by sex, and the population was categorized into 3 age groups: 0 to 54 years, 55 to 64 years, and 65 years and older. Age was calculated as a percentage, using eligibility data, and applied to the total member counts for the plan. We defined the care-seeking prevalence of MS by identifying the unique patients with an International Classification of Diseases Code-ninth revision (ICD-9) code 340 during the study period. ${ }^{11}$ Rates of disease treatment were measured by assessing how many patients had a pharmacy or medical claim using either national drug code (NDC) numbers or Health Care Procedural Coding System numbers (J-codes) that refer to the injectable treatments of IBlal (54569443300, 59627000103, J1825), IB1b (50419052101, $50419052105,50419052103,50419052115$, J1830), or glatiramer $(00088115003) .^{12,13}$

Patients were further stratified by new or existing patient status, as shown in Figure 1. The initial date of service in 2000 was compared with records from the previous 6 months to see if another diagnosis or treatment code indicating RRMS was present. If there was such a code, the patient was classified as an existing user. If there was no RRMS code, the patient was classified as a new user. The analysis assumed that the pharmacy benefit manager would process $100 \%$ of the prescription claims in this therapeutic class.

\section{Market-Share Projections}

The manufacturer of IBla2 provided us with projections for target markets estimating that IBla2 use among new MS patients 


\section{Pharmacy Benefit Forecast for a New Interferon Beta-1a for the Treatment of Multiple Sclerosis: Development of a First-Line Decision Tool for Pharmacy-Budget Planning Using Administrative Claims Data}

would be $80 \% .^{14}$ The manufacturer assumed that a shift to IBla2 among existing patients would depend on their current therapy, estimated at $65 \%$ for IBlal, 20\% for IB1b, and $25 \%$ for glatiramer users (Figure 1). The market-share for the base-case plan in 2000 was calculated as an overall percentage of days supply dispensed: $51 \%$ for IBlal, 34\% for IB1b, and 16\% for glatiramer.

Annual duration of therapy was used to estimate utilization per user. The mean sum of days supply dispensed under the pharmacy benefit in 2000 was calculated for current drug therapies. The resulting 253.13-day estimate (95\% CI, 235.89-270.36) was used to model similar duration of use by IB1a2 users.

\section{Benefit Design}

Four benefit-design scenarios were considered:

- Second formulary tier (\$15 copayment) with PA required

- Second formulary tier (\$15 copayment) with no PA

- Base case-third formulary tier (\$25 copayment) with PA required

- Third formulary tier (\$25 copayment) with no PA

The costs associated with administering a PA program were included in the analysis. Criteria for authorization for this drug would include confirming a diagnosis of relapsing multiple sclerosis. We estimated that $80 \%$ of PA requests would be approved. Of the denials, 50\% would be appealed and processed, and half of the appeals would be approved. PA review costs an estimated $\$ 30$ and appeals $\$ 45 .{ }^{15,16}$ (These costs are cumulative such that an initial review plus appeal would total $\$ 70 .{ }^{16}$ ) Treatment costs incurred by denied patients, based on the market share of the alternative injectable therapies, were also included, because the plan may have to pay for another treatment that is almost as expensive as IB1a2, and those costs would erode the savings accrued from the PA denial. The eventual acquisition of IB 1 a2 therapy through a PA override was also estimated at the rate of $14 \%$ of all denied patients. The base case assumed that, like the current treatments, the new therapy would be covered $100 \%$ under the pharmacy benefit, less the copayment amount.

\section{Costs of Therapy}

The $\$ 35.58$ estimated daily cost of therapy for IB1a2 was based on estimated wholesale acquisition cost (WAC) (80\% of average wholesale price for 1 month $\$ 1,334.13) .{ }^{17}$ This cost was then adjusted for migration from other therapies based on their respective market shares within the plan population.

For the base-case plan, we calculated the cost offset by multiplying the daily estimated WAC of each existing drug by its market share: for IBlal, $\$ 29.24$ multiplied by $51 \%$ (WAC effective January 10,2002 ); for IBlb, $\$ 30.05$ multiplied by $34 \%$ (WAC effective January 1, 2002); and for glatiramer, $\$ 27.71$ multiplied by $16 \%$ (WAC effective October 3, 2001). ${ }^{18}$ The resulting incremental cost of IBla2 was $\$ 6.02$ per day or

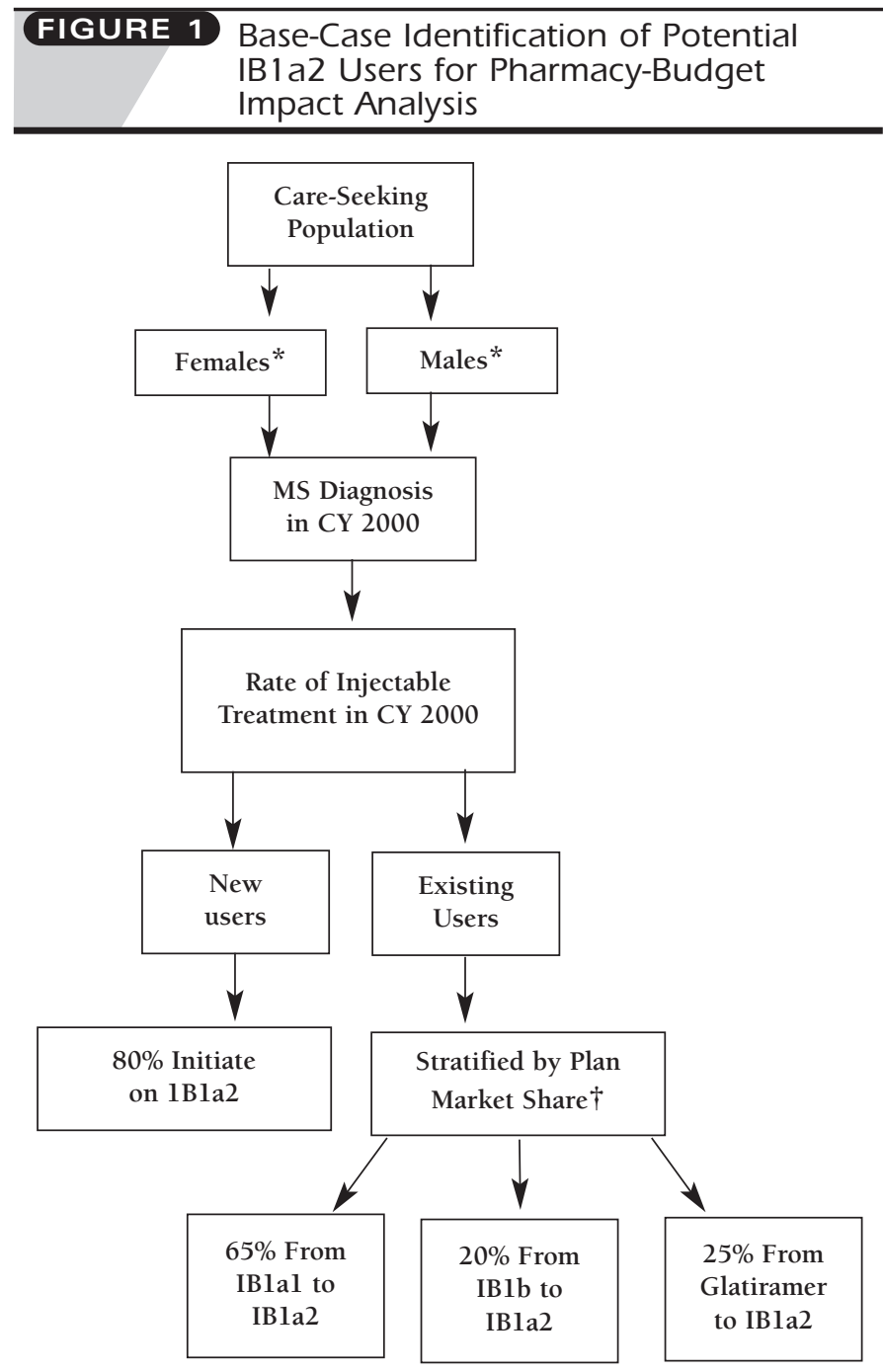

* Females and males were calculated separately. $\$ 51 \%$ IBlal, 34\% IBlb, 16\% glatiramer.

$\$ 1,523$ per average course of therapy.

The final cost calculation was based on the total number of patients approved to receive IBla2 (after the PA process) multiplied by the duration of therapy and the incremental daily cost of therapy. Total costs were adjusted to reflect PA administrative costs, savings from PA denials, eventual acquisition of IBla2 therapy, and the costs of alternative therapy for patients with PA denials. The costs were expressed using PMPM and per-member-per-year (PMPY) metrics.

\section{Market-Launch Curve}

Using the 2000 assessment of the total potential user popula- 


\begin{tabular}{lc}
\hline TABLE 2 & $\begin{array}{l}\text { Description of Base-Case Population } \\
\text { for Pharmacy-Budget Impact } \\
\text { Analysis of IB1 12 }\end{array}$ \\
\hline Characteristic & Value \\
\hline Plan Size* & 508,066 \\
\hline Number of members by age category (years) & $\mathbf{n ~ ( \% )}$ \\
0-54 & $365,808(72)$ \\
$55-64$ & $71,129(14)$ \\
$65+\dagger$ & $71,129(14)$ \\
\hline Female members by age category (years): & $\mathbf{n}(\%)$ \\
$0-54$ & $219,485(71)$ \\
55-64 & $42,678(14)$ \\
65+ & $46,945(15)$ \\
Coverage of injectables under pharmacy benefit & $100 \%$ \\
Risk for members over age 65 years & $100 \%$ \\
\hline
\end{tabular}

Calendar Year 2000 market share of existing

products based on days supply dispensed (\%):

IBlal

IBlb

Glatiramer

34

16

*All members eligible for pharmacy benefits as of December 31, 2000,

excluding the SCHIP program carrier.

$\dagger$ Counts of member population were based on calculated percentage of age applied to the current eligibility numbers. The $65+$ years population includes Medicare enrollees with supplemental coverage through the plan; i.e., does not include Medicare+Choice members.

\begin{tabular}{llllllll}
\hline TABLE 3 & $\begin{array}{l}\text { Estimates of Care-Seeking Behavior } \\
\text { for MS in Base-Case Health Plan by } \\
\text { Age and Sex (N=508,066) }\end{array}$ \\
\hline \multicolumn{6}{c}{ Males (\%) } \\
Characteristic & $0-54$ & $55-64$ & $65+$ & $0-54$ & $55-64$ & $65+$ \\
\hline MS diagnosis & 0.08 & 0.14 & 0.08 & 0.21 & 0.32 & 0.12 \\
\hline Injectable treatment rate & 44.62 & 20.00 & 11.00 & 51.80 & 42.65 & 6.90 \\
\hline New users & 40.38 & 12.50 & 0.00 & 33.05 & 18.97 & 0.00 \\
\hline Existing users & 59.62 & 87.50 & 100.00 & 66.95 & 81.03 & 100.00 \\
\hline Total predicted population (n) & 31 & 4 & 1 & 133 & 30 & 2 \\
\hline
\end{tabular}

tion, we profiled the phase-in of IBla2 for 3 years after market launch, assuming no growth in the RRMS market. The manufacturer's annual marketing targets were divided by calendar quarter and applied to the estimated PMPM. ${ }^{14}$

\section{Sensitivity Analysis}

One-way sensitivity analysis was performed to assess variation in the outcomes of

- duration of IBla2 therapy,

- migration of new users to IBla2, and

- shift of IBlal users to IBla2.

The model was developed as an interactive tool using
Microsoft Excel 97 and supplemented with data from claims analysis using Embarcadero RapidSQL version 5.5 or SPSS version 11.0. The spreadsheet can be modified for different health care payers by inserting specific demographics, benefit designs, and the market shares of existing RRMS treatments (IBlal, IBlb, and glatiramer).

\section{Results}

The demographic description of the study population is shown in Table 2. This plan had $72 \%$ of its membership under the age of 55 years; the remaining $28 \%$ of members were split equally between the 56 to 64 years and 65 years and older age categories. Sixty percent of the members aged 64 years and younger were female and $40 \%$ were male, while $66 \%$ of the members age 65 and older were female and 35\% were male. The basecase model settings for benefit design and coverage issues are shown in Table 3.

After the claims algorithm was applied (Figure 1), 201 individuals were considered candidates for IBla2 use. After applying rates of PA application, approval, and appeals, 146 individuals were identified as IBla2 users. The costs of therapy after a $\$ 25$ copayment per prescription were $\$ 191,498$. For the PA simulation, the costs were adjusted for PA administrative costs $(\$ 4,980)$, savings from PA denials in incremental IBla2 costs $(-\$ 18,363)$, annual costs of alternative therapies in denials $(\$ 104,725)$ and acquisition by some members receiving a PA override who never entered the PA process $(\$ 2,571)$. The total annual incremental cost for all patients in this 508,066 member plan seeking IBla2 in a third tier with PA scenario was $\$ 285,412$.

The estimated incremental impact of IB1a2 on the pharmacy budget for the base-case scenario was $\$ 0.047$ PMPM ( $\$ 0.56$ PMPY). The per-patient-per-year (PPPY) costs were estimated at $\$ 1,954.88$ or $\$ 162.91$ per-patient-per-month (PPPM). This represents the maximum additional drug expenditures after member cost share (i.e., the net additional cost for the drug plan sponsor) that could be expected in the RRMS class with the launch of IB1a2, assuming that all projected patients start using the drug. As shown in Figure 2, incremental PMPM was $\$ 0.053$ for patients aged 0 to 54 years, who comprise $72 \%$ of the study population. Although there are equal numbers of members in the remaining 2 categories, incremental PMPM for the 55 to 64 years category was $\$ 0.056$, compared with only $\$ 0.004$ for those aged 65 years and older.

The incremental PMPM impact by benefit design, shown in Figure 3, was calculated to be $\$ 0.049$ when IBla2 was placed on the second formulary tier and PA was required, compared with $\$ 0.046$ for tier 2 with no PA. The incremental PMPM is higher for the plan design with required PA due to PA administrative costs and the costs of alternative therapy for denied patients.

Estimated incremental PMPM for tier 3 was $\$ 0.047$ with PA and $\$ 0.043$ without PA. These estimates are lower than for tier2 plan designs, because patients pay a larger share of the cost, 


\section{Pharmacy Benefit Forecast for a New Interferon Beta-1a for the Treatment of Multiple Sclerosis: Development of a First-Line Decision Tool for Pharmacy-Budget Planning Using Administrative Claims Data}

which decreases the health plan's portion of the cost.

Sensitivity analysis was performed for IBla2 duration of therapy, IBla2 market share among new RRMS patients, and existing users' shift from IBlal to IBla2. As shown in Figure 4, the base-case estimates changed slightly when altering the duration of therapy by $25 \%$. The incremental budget impact was $\$ 0.035$ for 190 days' annual duration of therapy and $\$ 0.058$ for 316 days, compared with the base case of $\$ 0.046$ for 253 days. The model reacted less sensitively to the assumption that $80 \%$ of new users would initiate therapy on IBla2. Variation from $60 \%$ to $100 \%$ of the market share among new users caused a shift of only $\$ 0.04$ to $\$ 0.05$ in incremental PMPM. Changes in the projected market share from $49 \%$ to $81 \%$ of existing IBlal users to IBla2 similarly caused minimal fluctuation in the incremental PMPM (\$0.044 to \$0.049).

While the incremental increase in current drug expenditures for injectable MS therapy was less than $\$ 0.05$, Figure 5 shows that the estimated total cost for one year, after subtraction of member cost-share, for IBla2 one year after the market launch is $\$ 0.21$ PMPM for the base case, only slightly less than the current drug expenditures for IBlal (\$0.23).

Figure 6 shows the estimated 3-year market-launch curve for each product in the class and IBla2 uptake extrapolated based on manufacturer targets for market share. The estimated market share in year 3 is IBlal, 29.8\%; IBla2, 26.2\%; IBlb, 9.5\%; and glatiramer, $34.4 \%$. The estimated incremental PMPM after the IB1a2 launch is $\$ 0.02$ after year $1, \$ 0.03$ after year 2, and the $\$ 0.047$ at the end of year 3 .

\section{Discussion}

This model predicts that IBla2 will add an additional cost of $\$ 0.047$ PMPM to the pharmacy budget of a health plan with full injectable coverage and placement on tier 3 with PA required. Estimates of budget impact on the other 3 plan designs did not vary much from the base case after considering administrative fees and alternative therapy costs for PA denials. The predicted impact is considered low, relative to other therapies such as Cox-II inhibitors ${ }^{20}$ and allows for more flexible benefit design in the MS injectable drug class.

Although the estimates resulting from this study are specific to the age and sex distribution of the test health plan, the age of this population resembles the age distribution reported in the 1996 Medical Expenditure Panel Survey for the total insured population. ${ }^{19}$ The annual rates of MS prevalence derived from our study population are similar to those reported by Pope et al., ${ }^{6}$ using 2 years of claims data. Pope's prevalence rates were slightly lower for the 55 to 64 years age group (26/10,000 in Pope compared to $32 / 10,000$ here). ${ }^{6}$ We surmise that an increased percentage of females or a higher concentration of members aged 35 to 54 years would increase estimates of the potential population of users. Accordingly, we would anticipate budget impact to be higher in these scenarios as well.

The most significant driver in the model was duration of
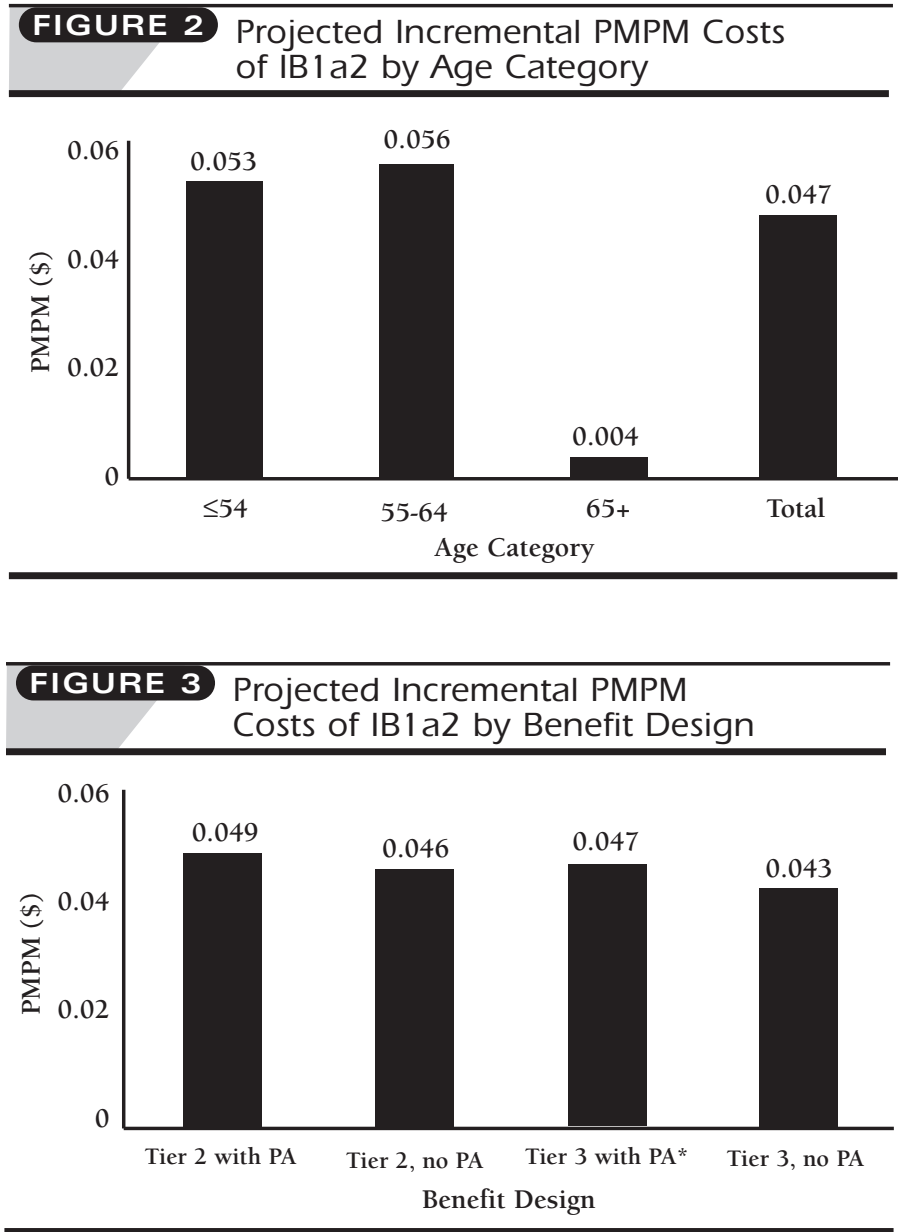

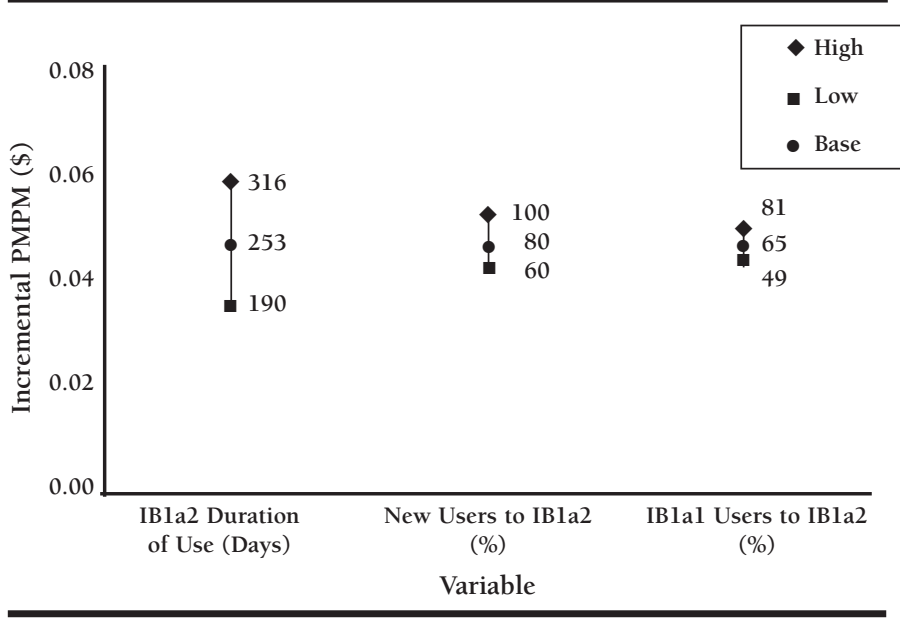

IB1a2 therapy. Increasing the average duration of therapy by $25 \%$ increased the estimated incremental PMPM to $\$ 0.058$ (\$0.70 PMPY). Since duration of therapy was derived from 

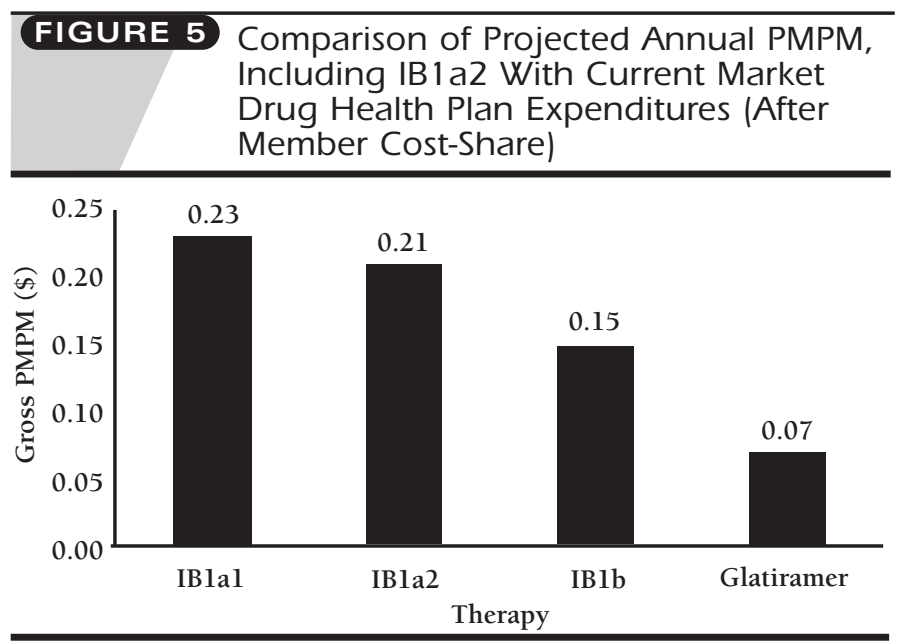

FIGURE 6 Market-Launch Curve With Incremental PMPM Shift for 3 Years Postlaunch

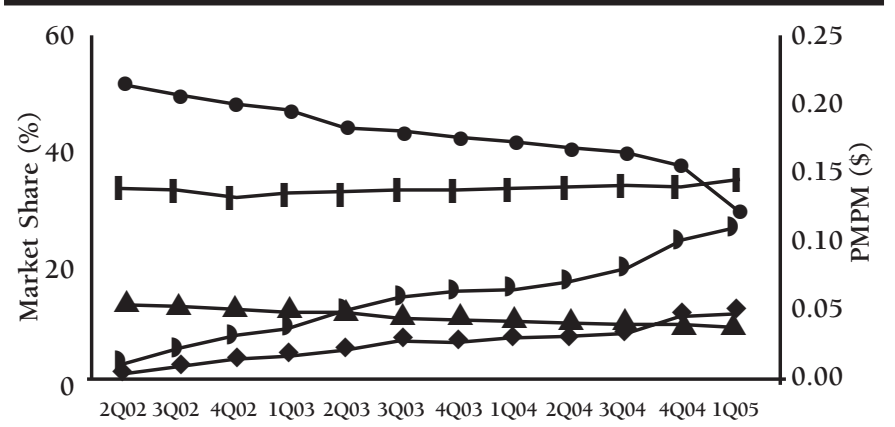

Time

- Ibla1 IIbla2 $\triangle$ IB1b $\quad$ Glatiramer $\quad$ IBla2 Incremental PMPM

actual patient experience with similar competitors in this therapeutic class, we used that as a surrogate marker for compliance. Clinical trial data suggests that increased efficacy and lower side-ffect profiles can be achieved by IBla2 therapy. ${ }^{2}$ A byproduct of these improvements may be increased patient compliance, which could raise the average duration of therapy and ultimately affect the incremental cost. However, the predicted change would be small ( $\$ 0.047$ to $\$ 0.058)$ and potentially offset by medical savings due to improved efficacy.

This analysis used fixed pricing for all of the agents considered since the price at launch had already been determined. Additionally, the new agent was already priced at higher cost than the other agents on the market and would most likely not stimulate price reduction in the competition. If the incremental price of the agent was twice what it is today ( $\$ 12$ per day), the incremental budget impact would be $\$ 0.08$ PMPM. Ideally, budget impact analyses should consider price of the agents and potential changes in the market as competitors react to market changes. If the price of the competitor agents decreased without a parallel price decrease in Ibla2, the budget impact would be higher. If the price of the new agent was reduced without a price shift in the competitors, the impact of the new agent would be smaller. Inflationary pressures over time would most likely affect each competitor similarly, and the incremental impact would remain the same.

The manufacturer had predicted a high percentage-80\%of IBla2 market penetration among new users similar to the addition of pegylated interferon for the treatment of hepatitisC. ${ }^{21}$ We felt this utilization target was high (making it conservative from a pharmacy budget standpoint) and performed sensitivity analysis around that assumption. However, 20\% variation in this percentage did not greatly affect the PMPM estimate due to the small number of patients affected by this market-share estimate.

Our analysis was undertaken to develop a method for estimating budget impact that could be readily customized to individual health plan needs. For instance, prevalence of the disease is an important consideration since it may vary by plan. A doubling of the number of patients identified in this scenario would increase the base-case incremental impact to \$0.09 PMPM. A $50 \%$ reduction in the number of patients results in an incremental impact of $\$ 0.02$ PMPM. While these are relatively small increases, they do reflect the need for health plans to have customized information. Administrative claims data can provide a ready source for such customization.

The model assumes that there would be no additional growth in the number of diagnosed cases of RRMS, so the number of patients per year would be relatively static. If new diagnostic tests or widespread education about RRMS promotes more care-seeking behavior, there may be unanticipated growth in the market. However, any increased incidence would not be solely attributed to the launch of IBla2, and the relative magnitude of the impact to the therapeutic class would likely remain the same. Future drug releases or market shifts could also impact the market-launch curve as we have predicted it.

This analysis assumes that a health plan is at risk for the administrative costs associated with conducting PA programs. While this is true for many health plans, some insurers may shift that cost to their pharmacy benefit manager (PBM). The rates of PA denials and appeals were derived from PBM experience with Cox-2 inhibitors, so actual experience with this new injectable therapy may differ. However, widespread denial of this drug to treat a serious, debilitating condition is unlikely.

While claims data may represent a better estimate of the care-seeking behavior in specific communities, selection bias and coding errors may affect the results. For instance, a 1-year cross-sectional analysis of ICD-9 codes may underestimate chronic conditions that are not relevant with each physician visit. Also, the use of an employer-group dataset biases the test population toward healthy workers. It may not be appropriate to apply this analysis to Medicaid or Medicare populations with 


\section{Pharmacy Benefit Forecast for a New Interferon Beta-1a for the Treatment of Multiple Sclerosis: Development of a First-Line Decision Tool for Pharmacy-Budget Planning Using Administrative Claims Data}

higher disease prevalence; accurate results would most likely require different assumptions.

Costs associated with side effects or adverse events that may require other drug therapies are not included in the model. The current clinical trial data do not suggest that IBla2 would cause a disproportionate number of adverse events that would require additional drug therapy and thereby raise the incremental costs. Conversely, if the side-effect profile is improved with IBla2, use of the new agent may reduce the impact on the pharmacy budget.

Future development of budget forecast analyses should include a wider range of benefit design options that are increasingly used in health plans, including percentage copayments, 4 or 5 copayment tier placement, and defined contribution plans. Methods should also be developed to validate the findings in specific populations after a forecast analysis has been implemented and a new drug launch can be evaluated. Deficits in the accuracy of models can be addressed in future simulations. While budget impact modeling is necessary and relevant, the technique relies on inexact science of cost calculations and assumptions.

The strength of this pharmacy budget impact approach is that rates of utilization are based on actual community data. The pharmacy-budget payer's perspective was aided by providing useful metrics. The analysis is a first-line decision tool for benefit design and helps direct the agenda for further pharmacoeconomic research that many include broader medical-cost implications.

\section{Conclusion}

IB1a2 will have minimal incremental budget impact $(\$ 0.047$ PMPM for third tier with prior authorization) over the current drug expenditures for the drug plan sponsor (i.e., after member cost-share) for RRMS therapies. This pharmacy-budget impact analysis indicates that health care benefit managers have flexibility when designing coverage for IBla2 and can emphasize choice and clinical efficacy over cost containment.

\section{ACKNOWLEDGMENT}

Devora Mitrany, ELS, an employee of AdvancePCS, assisted with the preparation of this manuscript.

\section{DISCLOSURES}

Serono Incorporated provided funding for the completion of this analysis and manuscript. Author Robert Phipps is employed by Serono, manufacturer of interferon beta-la. Support was also provided by AdvancePCS. Funding was obtained by author Alan Wright. Author Christina M. Meyer served as principal author of the study. Study concept and design was the work of Meyer, Wright, Phipps, and author Deborah Cooper. Analysis and interpretation of data was the work of Meyer, Cooper, Wright, and Phipps. Meyer contributed statistical expertise. Drafting of the manuscript was the work of Meyer and its critical revision was the work of Cooper, Phipps, and Wright. Administrative, technical, and/or material support was provided by Devora Mitrany.

\section{REFERENCES}

1. U.S. Food and Drug Administration Center for Drug Evaluations and Research. Department of Health and Human Services, Orphan Products Development. Memorandum: March 7, 2002. Analysis of exclusivity issues raised in the Serono BLA for Rebif. Available at: http:// www.fda.gov/cder/htm. Accessed March 8, 2002.

2. Färkkilä M. The EVIDENCE study-direct comparative study of IFN Beta la in RRMS [abstract]. 17th Congress of the European Committee for Treatment and Research of Multiple Sclerosis; September 12-15, 2001; Dublin, Ireland. In press.

3. Serono Corporation press release, March 8, 2002. Available at: http://www.serono.com/media/index.htm. Accessed March 8, 2002.

4. Nuijten MJC, Hutton J. Cost-effectiveness analysis of interferon beta in multiple sclerosis: a Markov process analysis. Value Health. 2002;5(1):44-54.

5. National Multiple Sclerosis Society. Available at: http://www.nationalmssociety.org/What\%20is\%20MS.asp. Accessed February 3, 2003.

6. Pope GC, Urato CJ, Kulas ED, et al. Prevalence, expenditures, utilization and payment for persons with MS in insured populations. Neurology. 2002;58:37-43.

7. Trueman P, Drummond M, Hutton J Developing guidance for budget impact analysis. Pharmacoeconomics. 2001;19(6):609-21

8. Bootman JL, Townsend RJ, McGhan WF. Principles of Pharmacoeconomics. Cincinnati, Ohio: Harvey Whitney Books Company; 1996:60-75.

9. Siegel JE, Torrance GW, Russell LB, et al. Guidelines for pharmacoeconomic studies: recommendations from the panel on cost-effectiveness in health and medicine. Pharmacoeconomics. 1997 Feb;11(2):159-68.

10. Centers for Medicaid and Medicare Services. State Children's Health Improvement Program. Available at: http://cms.hhs.gov/schip/. Accessed February 10, 2002.

11. Medicode 2002 ICD-9CM Expert for Hospitals. Vol. 1, 2, and 3. 6th ed. West Valley City, Utah: St. Anthony Publishing; 2001.

12. Food and Drug Administration Center for Drug Evaluation and Research NDC Download List. Available at: http://www.fda.gov/cder/ndc/. Accessed February 1, 2002.

13. Centers for Medicaid and Medicare Services. 2002 Alpha-numeric files for Healthcare Common Procedure Coding System. Available at: http://cms.hhs.gov/medicare/hcpcs/default.asp. Accessed February 1, 2002.

14. Personal communication, vice president of managed markets, Serono Incorporated.

15. Reissman D. What is the real cost of prior authorization? Drug Benefit Trends. 2000; 12(10):22,24

16. Utilization Management AdvancePCS. Data on file.

17. Drug Topics Red Book. Montvale, NJ: Thompson Medical Economics; 2001:April 2002 update, page 10.

18. Drug Topics Red Book. Montvale, NJ: Thompson Medical Economics; 2001. 19. Center for Cost and Financing Studies, Agencies for Healthcare Research and Quality. Medical Expenditure Panel Survey: Household Component, 2000.

20. Acid blocker use has not declined following Celebrex launch, PBM finds. Pink Sheet. June 7, 1999;61(23):12.

21. Personal communication, financial analyst, AdvancePCS. Data on file. 